\title{
Bone Marrow Mesenchymal Stem Cells Inhibit Lipopolysaccharide-Induced Inflammatory Reactions in Macrophages and Endothelial Cells
}

\author{
Dequan Li, ${ }^{1}$ Cong Wang, ${ }^{2}$ Chuang Chi, ${ }^{3}$ Yuanyuan Wang, \\ Jing Zhao, ${ }^{4}$ Jun Fang, ${ }^{1}$ and Jingye Pan $^{4}$ \\ ${ }^{1}$ Department of Traumatology Medicine, The First Affiliated Hospital of Wenzhou Medical University, Wenzhou, \\ Zhejiang 325000, China \\ ${ }^{2}$ School of Pharmacy, Wenzhou Medical University, Wenzhou, Zhejiang 325000, China \\ ${ }^{3}$ Department of Cardiothoracic Surgery, The First Affiliated Hospital of Wenzhou Medical University, Wenzhou, \\ Zhejiang 325000, China \\ ${ }^{4}$ Department of Intensive Care Unit, The First Affiliated Hospital of Wenzhou Medical University, Wenzhou, \\ Zhejiang 325000, China
}

Correspondence should be addressed to Jingye Pan; panjingye@wzhospital.cn

Received 31 August 2015; Revised 24 December 2015; Accepted 6 January 2016

Academic Editor: Teresa Zelante

Copyright ( 2016 Dequan Li et al. This is an open access article distributed under the Creative Commons Attribution License, which permits unrestricted use, distribution, and reproduction in any medium, provided the original work is properly cited.

\begin{abstract}
Background. Systemic inflammatory response syndrome (SIRS) accompanied by trauma can lead to multiple organ dysfunction syndrome (MODS) and even death. Early inhibition of the inflammation is necessary for damage control. Bone marrow mesenchymal stem cells (BMSCs), as a novel therapy modality, have been shown to reduce inflammatory responses in human and animal models. Methods. In this study, we used Western blot, quantitative PCR, and enzyme-linked immunosorbent assay (ELISA) to assess the activity of BMSCs to suppress the inflammation induced by lipopolysaccharide (LPS) in human umbilical cord endothelial cells (HUVECs) and alveolar macrophages. Results. Our results demonstrated that LPS caused an inflammatory response in alveolar macrophages and HUVECs, increased permeability of HUVEC, upregulated expression of toll-like receptor (TLR) 2, TLR4, phosphorylated p65, downregulated release of IL10, and promoted release of TNF- $\alpha$ in both cells. Coculture with BMSCs attenuated all of these activities induced by LPS in the two tested cell types. Conclusions. Together, our results demonstrate that BMSCs dosage dependently attenuates the inflammation damage of alveolar macrophages and HUVECs induced by LPS.
\end{abstract}

\section{Introduction}

Traumatic injury is the leading cause of morbidity and mortality, which affects many parts of the body, including the brain, extremities, and internal organs. The incidence of lifethreatening complications, such as systemic inflammatory response syndrome (SIRS) and acute lung injury (ALI), in severely injured trauma patients remains between $30 \%$ and $50 \%[1,2]$. SIRS induced by severe trauma or other injuries is a clinical syndrome initiated by dysregulation of inflammation, which could lead to various tissue injuries culminating in multiple organ dysfunction/failure syndrome (MODS/MOF).
Bone marrow-derived mesenchymal stem cells (BMSCs), also referred to as marrow stromal cells, are a multipotential lineage characterized by the capacity for extracorporeal expansion and the ability to differentiate into bone, cartilage, and adipose tissues. BMSCs appear to function as potent immunomodulators. Numerous studies indicated that BMSCs can be an effective modality for cell-based immunomodulatory therapies in various diseases, such as sepsis, endotoxemia, diabetes, and lung injury [3-9], especially for reducing of inflammation $[4,5,10]$. Several clinical trials assessing the efficacy of BMSCs in immune-mediated diseases are currently underway. However, how BMSCs attenuate inflammatory reaction is poorly understood. 
Quick and efficient response to microbial infections is driven by recognition of molecules broadly shared by a variety of pathogens, which are distinguishable from host molecules named pathogen associated molecular patterns (PAMPs). Pattern-recognition receptors (PRRs) that contain membrane-bound PRRs, including toll-like receptors (TLRs) and other cytoplasmic proteins, recognize the PAMPs [10]. Upon binding with the PAMPs, TLR2 and TLR4 then activate NF- $\kappa \mathrm{B}$ and subsequently promote cytokine synthesis, which include IL-1, IL-6, and TNF- $\alpha[11,12]$. The network of TLRs and PRRs mediates the response of BMSCs to inflammatory stimuli, such as LPS $[13,14]$. However, how TLRs modulate the BMSC activity is not clearly elucidated. Since TLR2 and TLR4 response to acute otitis through activation of $\mathrm{NF}-\kappa \mathrm{B}[15]$, we hypothesized that BMSCs ameliorate the inflammation damage of alveolar macrophages and HUVECs through the inhibition of TLR2 and TLR 4 mediated NF- $\kappa \mathrm{B}$ pathways.

\section{Materials and Methods}

2.1. Cell Culture. Alveolar macrophages and HUVECs were purchased from Chi Scientific, Inc. (China). The cells were cultured in RPMI 1640 medium (Sigma Chemical Co., St. Louis, MO) supplemented with $10 \%$ fetal bovine serum (FBS) (Gibco, Carlsbad, CA, USA) and 1\% penicillin/streptomycin (Solarbio, Beijing, China) at $37^{\circ} \mathrm{C}$ in a humidified atmosphere of $5 \% \mathrm{CO}_{2}$. BMSCs obtained from Cyagen Biosciences Inc. (Suzhou, China) were cultured in 10\% FBS-DMEM-LG (Gibco, Carlsbad, CA, USA). The surface markers for BMSCs differentiation of adipocytic, osteogenic, and chondrogenic lineages were characterized as previously reported [16]. Only passages 3-7 of the cells were used for experiments.

2.2. Endothelial Permeability Assay. Monolayer permeability was quantitated by spectrophotometric measurement of the flux of Evans blue-albumin across HUVECs as described previously [17]. HUVECs $\left(1 \times 10^{5}\right)$ were seeded in 24-well Transwell inserts $(5 \mu \mathrm{m}$ pore size, Corning Incorporated, NY, USA) and cultured with 10\% FBS DMEM overnight and then changed with serum-free medium for another 24 hours. The various numbers of BMSCs were seeded at the bottom of the same 24-well plate with serum-free media. LPS was added to the Transwell inserts at a final concentration of $100 \mathrm{ng} / \mathrm{mL}$ for different time points $(1,3,6,12$, and $24 \mathrm{~h})$. After that, the BMSCs were digested by trypsin-EDTA solution to terminate the effects of BMSCs and $100 \mu \mathrm{L}$ Hank's solution containing Evans blue- (EB-) conjugated bovine serum albumin (final concentration $0.67 \mathrm{mg} / \mathrm{mL}$ ) was added to the Transwell inserts, while $600 \mu \mathrm{L} 4 \%$ BSA was added to the lower plate chamber. After incubation at $37^{\circ} \mathrm{C}$ for 1 hour, $100 \mu \mathrm{L}$ BSA solution from the lower chamber was harvested and measured for the absorbance at $620 \mathrm{~nm}$. The HUVECs permeability capacity was calculated as follows: Evans blue dye-labeled albumin (EB-albumin) leak rate = OD620 $0_{\text {leaked EB-albumin }} /$ OD620 $0_{\text {total EB-albumin }} \times 100 \%$.

2.3. Alveolar Macrophages/HUVECs-BMSC Coculture Experiments. Alveolar macrophages or HUVECs $\left(1 \times 10^{6} /\right.$ well $)$ were seeded in a 6-well plate, after 24-hour incubation, BMSCs $\left(2 \times 10^{5} /\right.$ well $)$ were seeded in the 6-well Transwell inserts ( $5 \mu \mathrm{m}$ pore, Corning Incorporated, NY, USA) at the indicated time points $(1,3,6,12$, and $24 \mathrm{~h})$. LPS $(100 \mathrm{ng} / \mathrm{mL})$ was then added to the lower chamber for $1 \mathrm{~h}$. Alveolar macrophages or HUVECs lysate was collected for RNA and protein analyses, and the cell-free supernatants were collected for ELISA detection.

2.4. Western Blot Analysis. Alveolar macrophages/HUVECs were lysed in the RIPA buffer (Thermo Scientific, Rockford, USA) supplemented with $1 \%$ PMSF and $1 \%$ protein phosphatase inhibitor mixture (P1260, Applygen, Beijing, China). The concentration of the proteins was measured with the Bicinchoninic Acid Kit (Thermo Scientific, Rockford, USA). Rabbit anti-TLR2, TLR4, and phosphorylated p65 unit of NF$\kappa \mathrm{B}$ antibodies were obtained from Cell Signaling Technology. Horseradish peroxidase- (HRP-) conjugated goat antirabbit antibodies were purchased from Bio-Rad. ECL-Plus Chemiluminescent Reagent (Thermo Scientific, Rockford, USA) was used to visualize the specific proteins. Relative concentration of proteins was quantitated using the Image J Software (National Institutes of Health, Bethesda, USA).

2.5. Gene Expression Analysis. Total RNA was isolated from cells using the TRIzol RNA Isolation Reagents (Life Technologies). The first-strand cDNAs were converted using the SuperScript III reverse transcriptase (Invitrogen, Carlsbad, CA) according to the manufacturer's protocols. Real-time RT-PCR analysis was performed on ABI 7500 Sequence Detection System using the UltraSYBR Mixture (CW Bio Co. Ltd, Beijing, China). Transcript expression was normalized with the glyceraldehyde-3-phosphate dehydrogenase (GAPDH) housekeeping gene. The PCR primer sequences are GAPDH forward 5'-TGGAGTCTACTGGCGTCTT$3^{\prime}$, reverse $5^{\prime}$-TGTCATATTTCTCGTG GTTCA-3'; TLR4 forward $5^{\prime}$-CTGCATAGAGGTAGTTCCT-3', reverse $5^{\prime}$ TCCAGCC ACTGAAGTTCTGA-3'; TLR2 forward $5^{\prime}$ GGAGACTCTGGAAGC ATG-3', reverse $5^{\prime}$-GCATCCT GAAGCCTGTG-3'.

2.6. ELISA Analysis. The supernatants collected from coculture system were centrifuged for 10 minutes at $1000 \mathrm{~g}$ $\left(4^{\circ} \mathrm{C}\right)$ to remove cell debris. The levels of TNF- $\alpha$ and IL10 were measured using commercially available enzyme-linked immunosorbent assay (ELISA) kits (BD Biosciences, San Diego, CA, USA) according to the instructions provided by the manufacturer.

2.7. Statistical Analysis. SPSS 17.0 software was used to perform the statistical analysis and values are presented as the means \pm standard deviations (SD); all experiments were repeated at least three times. The one-way ANOVA was used to determine the significance of differences between various groups. $P<0.05$ was considered to be statistically significant.

\section{Results}

3.1. BMSCs Inhibit LPS to Induce Hyperpermeability in HUVECs. To assess how BMSCs affected HUVECs permeability induced by LPS, HUVECs with or without coculture 


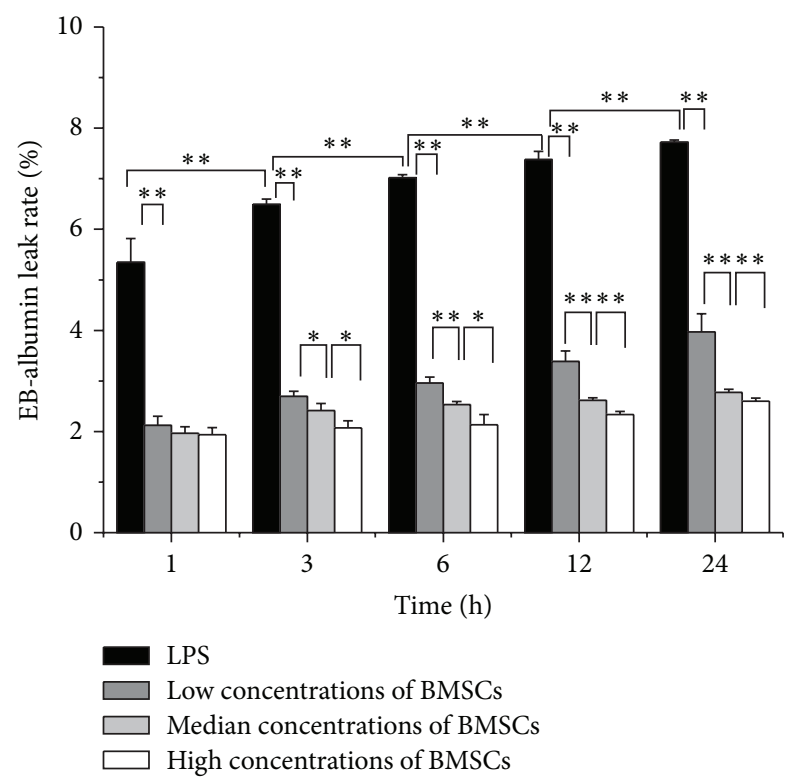

FIGURE 1: Effect of BMSCs on the barrier permeability of HUVECs. HUVECs were stimulated by LPS at a dosage of $100 \mathrm{ng} / \mathrm{mL}$ (black bars). In addition, HUVECs were cocultured with indicated concentrations of BMSCs: low $\left(1 \times 10^{3}\right.$ cells $/ \mathrm{mL}$, dark grey bars $)$, median $(1$ $\times 10^{4}$ cells $/ \mathrm{mL}$, light grey bars $)$, and high $\left(1 \times 10^{5}\right.$ cells $/ \mathrm{mL}$, white bars) for $1,3,6,12$, and $24 \mathrm{~h}$ followed by measuring permeability as described in Section 2. All results are means \pm SD of at least three different experiments. ${ }^{*} P<0.05 ;{ }^{* *} P<0.01$.

with BMSCs were stimulated by LPS for the indicated times. Without coculture with BMSCs, the EB-albumin leaking rate ranged from $5.35 \%$ (at $1 \mathrm{~h}$ ) to $7.72 \%$ (at $24 \mathrm{~h}$ ) in the LPS group (Figure 1). Compared with the LPS group, the EB-albumin leaking rates in the BMSC coculture group were reduced in a BMSC cell number- and time-dependent manner. The EB-albumin leaking rate in the $1 \times 10^{4} / \mathrm{mL}$ BMSCs group was decreased 3 hours after coculture. The effects were more significant after coculture for 6 hours to 24 hours. The effects were less significant in the group with $1 \times 10^{3} / \mathrm{mL}$ BMSCs and more significant in the group with $1 \times 10^{5} / \mathrm{mL}$ BMSCs (Figure 1).

3.2. BMSCs Attenuate LPS-Induced Expression of TLR2, TLR4, and p65 in Alveolar Macrophages and HUVECs. To determine whether LPS stimulated expression of TLR2, TLR4, and p65 in macrophages and HUVECs was affected by BMSCs, alveolar macrophages and HUVECs cells were cocultured with BMSCs for the indicated time followed by LPS treatment. Western blot (Figure 2) and real-time RT-PCR analyses (Figure 3) showed that LPS administration increased expression of TLR2, TLR4, and p65 within 1 hour after the treatment. The effect persisted even until 24 hours after the treatment. However, the activity of LPS was attenuated by coculture with BMSCs in both alveolar macrophages and HUVECs (Figures 2 and 3). Release IL10 and TNF- $\alpha$ in Response to LPS Stimulation. To determine whether BMSCs regulated the release of TNF$\alpha$ and IL10 from alveolar macrophages and HUVECs in response to LPS stimulation, the conditioned medium was collected from the coculture system and the concentrations of IL10 and TNF- $\alpha$ were assessed by ELISA. It was clear that concentration of TNF- $\alpha$ in the medium was increased after LPS stimulation within 1 hour and the effects lasted for 24 hours. However, the increases were blunted by coculture with BMSCs. In contrast, IL10 was reduced by LPS stimulation, while coculture with BMSCs increased medium IL10 production. The results indicate that BMSCs suppress LPSinduced inflammation by decreasing TNF- $\alpha$ and increasing IL10 releases (Figure 4).

\section{Discussion}

BMSCs treatments have been shown to reduce inflammatory response in human and animal models in response to injury. However, the detailed mechanism underlying this effect is not clear. Here, we reported that BMSCs alleviated HUVEC damage induced by LPS. The results also showed that BMSCs restricted the inflammatory responses of HUVECs and alveolar macrophage stimulated by LPS and therefore protected HUVECs from damage induced by the endotoxin. The data indicate that attenuating LPS-induced TNF- $\alpha$ pathway activation in HUVECs and macrophage by BMSCs contributes to the protection of HUVECs from endotoxin-induced damage by BMSCs.

Our data demonstrated that LPS increased the permeability of HUVECs monolayers in a time-dependent manner. Although the extent of the activity was lower than that after coculture for a longer time, the protection effects were detectable within one hour. The underlying mechanism of the protection is not clear. Since BMSCs and HUVECs were cultured separating by the Transwell membrane, the effects of BMSCs must be mediated by soluble factors released from BMSCs. However, we did not rule out the possibility that BMSCs sequestrated LPS and therefore protected the endothelial cells. Future efforts are needed to identify these beneficial secretory factors from BMSCs. Although LPS still induced HUVECs damage in the coculture, the EB-albumin leaks were reduced compared with the group without coculture with BMSCs. This suggests that BMSCs attenuate the damage. We observed that the protective effect of BMSCs on HUVEC was cell number-dependent. High concentrations of BMSCs were more effective than low concentrations of BMSCs. It appeared that the BMSCs released sufficient protective molecules to protect endothelial cells within one hour. Therefore, immediately administration of BMSCs to SIRS patients shall have beneficial effects. Furthermore, the results were in line with previous reports that LPS leads to persistent damage of HUVECs $[18,19]$. However, it was different from the report that the damage of HUVEC by LPS is not time-dependent [17]. The discrepancy is likely due to different culture time. We cultured the cells for 24 hours to reach the confluence, followed by serum starvation for 24 hours and LPS treatment. This method is different from the published one where the cells were cultured for 4 days prior 


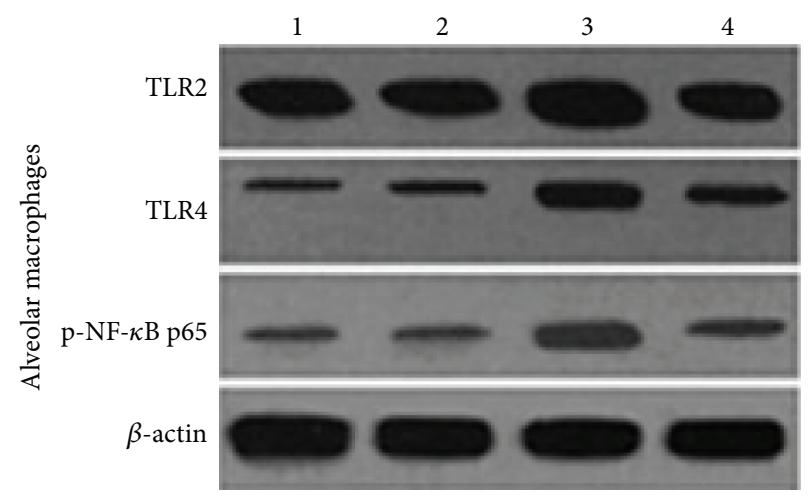
(1) PBS control
(2) BMSCs control
(3) LPS
(4) BMSCs treatment

(A)

TLR2

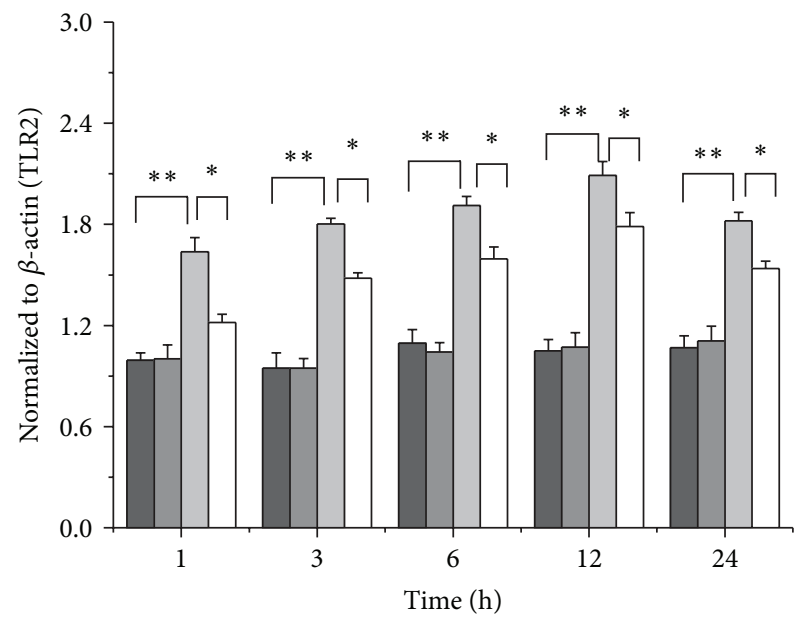

$\square$ PBS control BMSCs control $\square$ LPS

$\square$ BMSCs treatment

(B)

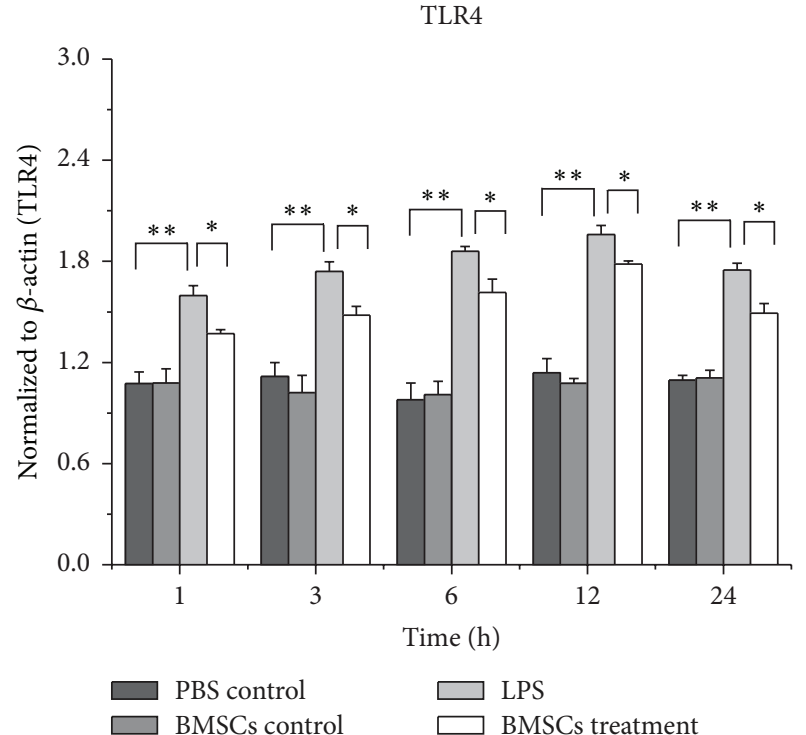

(C)

p-NF- $\kappa$ B p 65

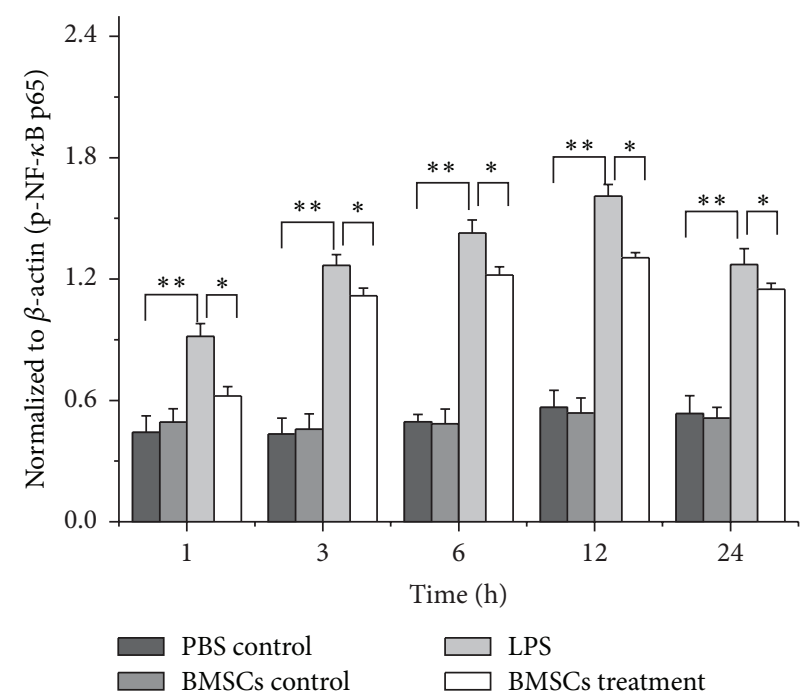

(D)

(a)

Figure 2: Continued. 


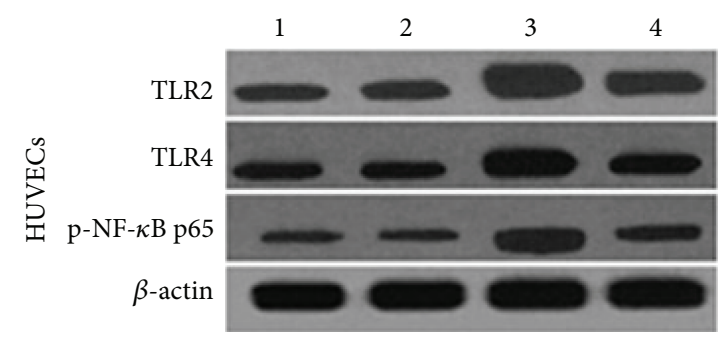
(1) PBS control
(2) BMSCs control
(3) LPS
(4) BMSCs treatment

(E)

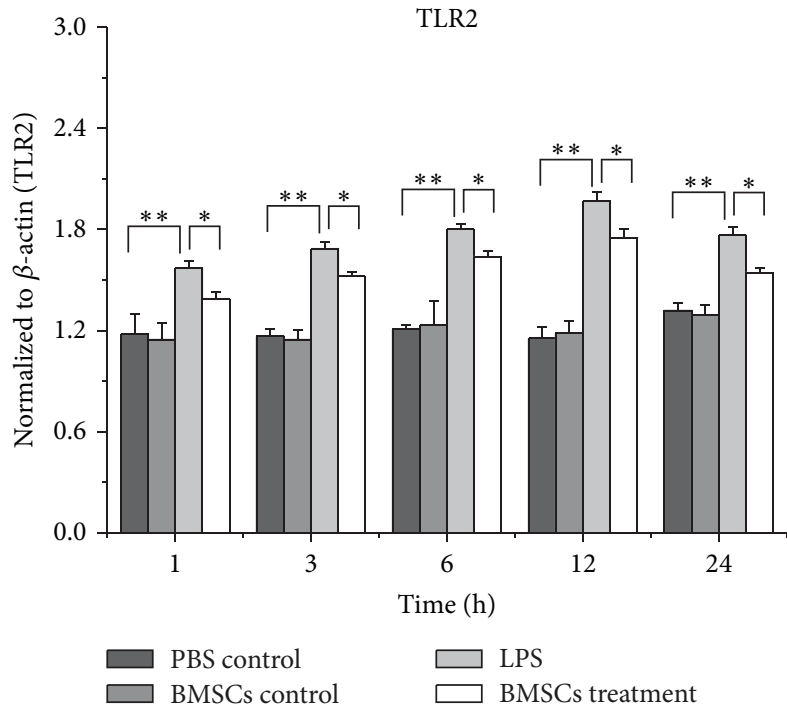

(F)

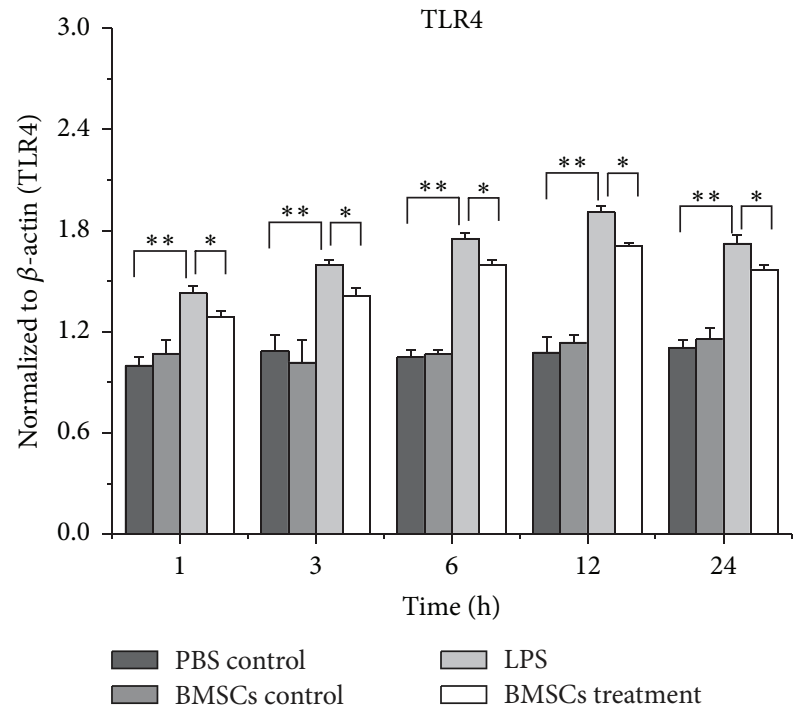

(G)

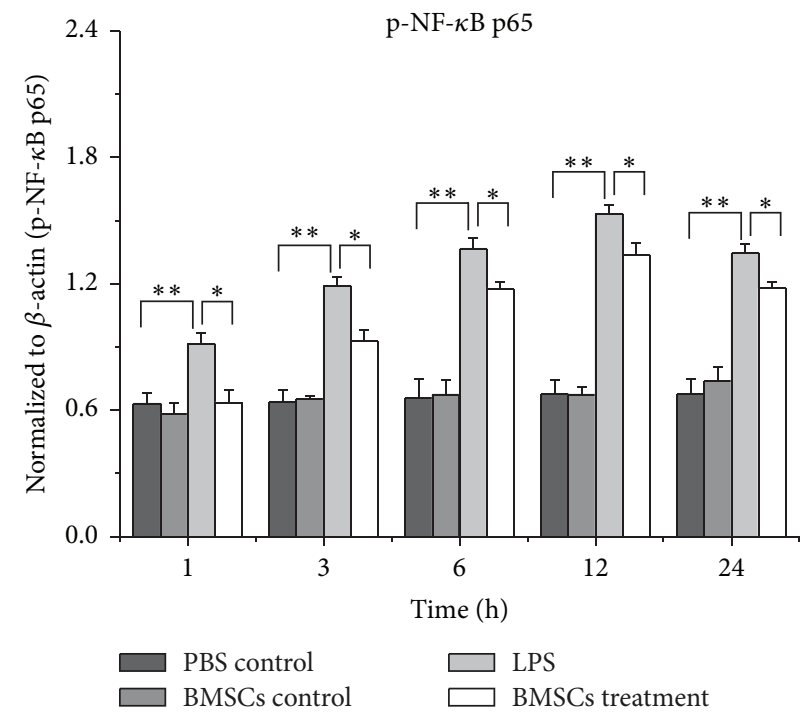

(H)

(b)

FIGURE 2: Coculture with BMSCs attenuates the activity of LPS to induce expressions of TLR2, TLR4, and p65 in alveolar macrophages (a) and HUVECs (b) at the protein level. The total proteins were extracted from the cultured alveolar macrophages and HUVECs treated (1) PBS control, (2) BMSCs, (3) LPS, and (4) BMSCs and LPS. The expressions of TLR2, TLR4, and p-NF- $\kappa$ B p65 were determined by Western blot. The density of specific bands was quantitated. Data are expressed as the mean \pm SD from three experiments. ${ }^{*} P<0.05,{ }^{* *} P<0.01$. 


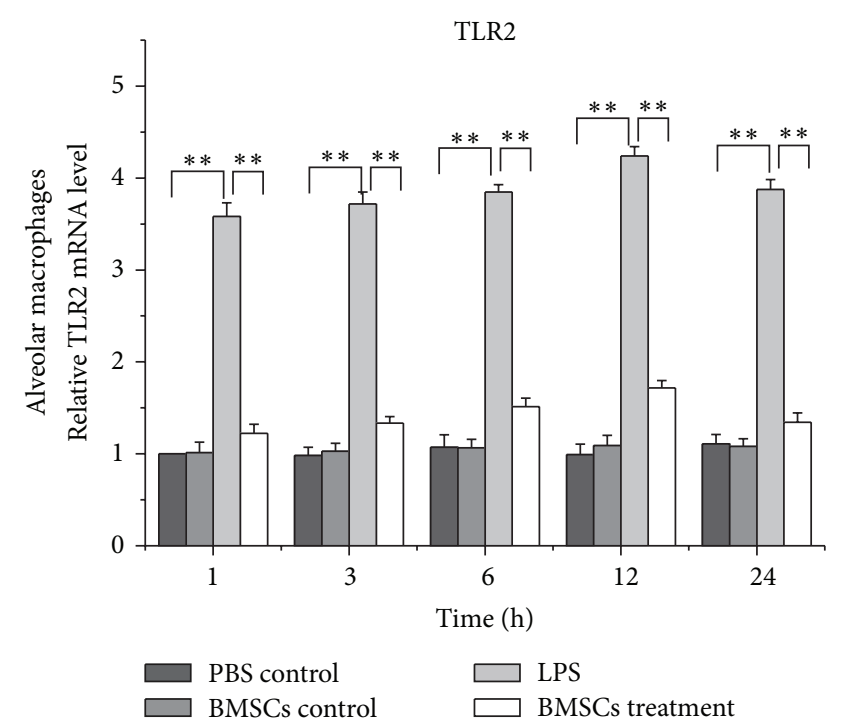

(A)

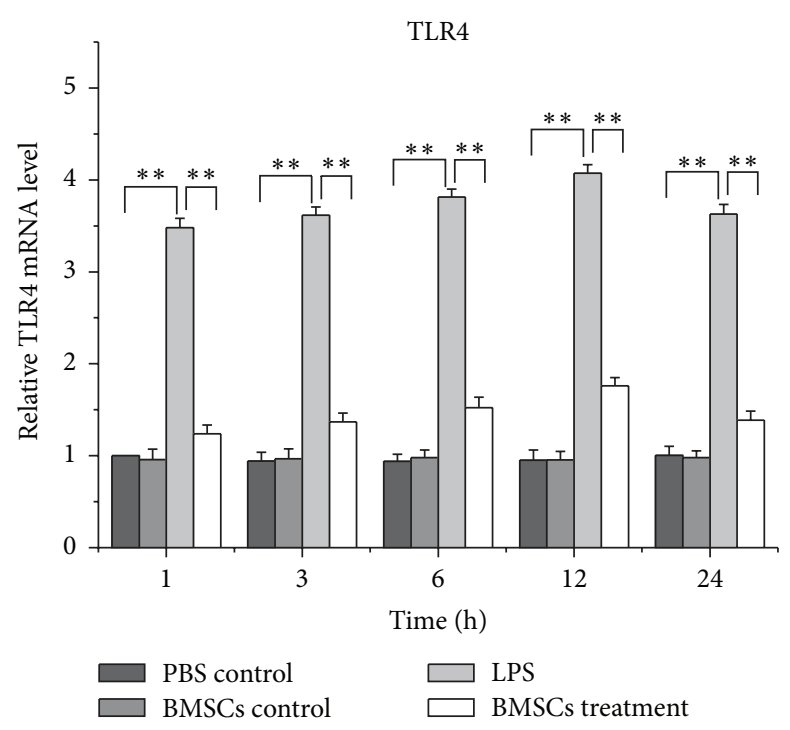

(B)

(a)

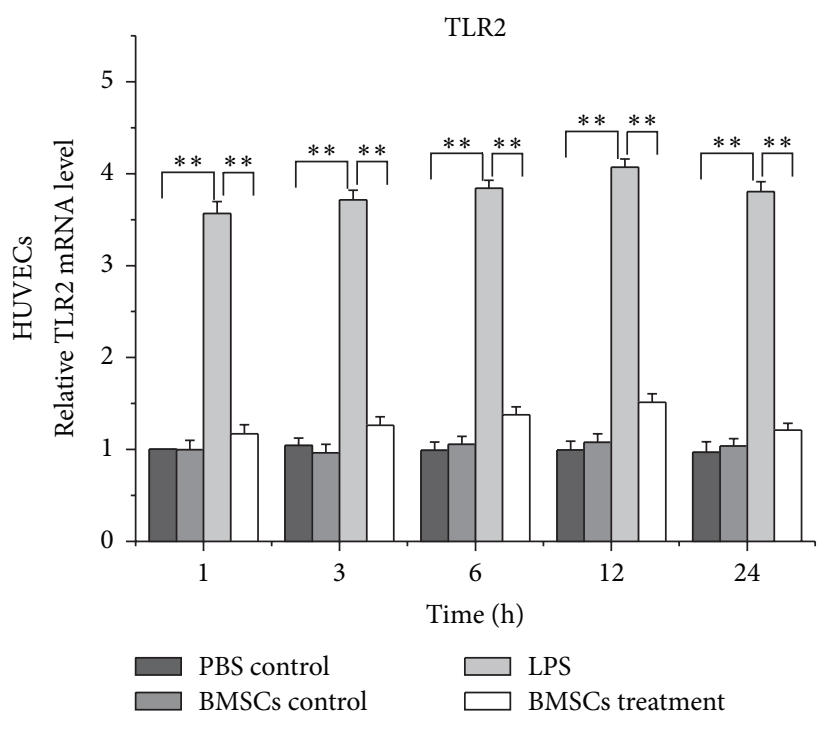

(C)

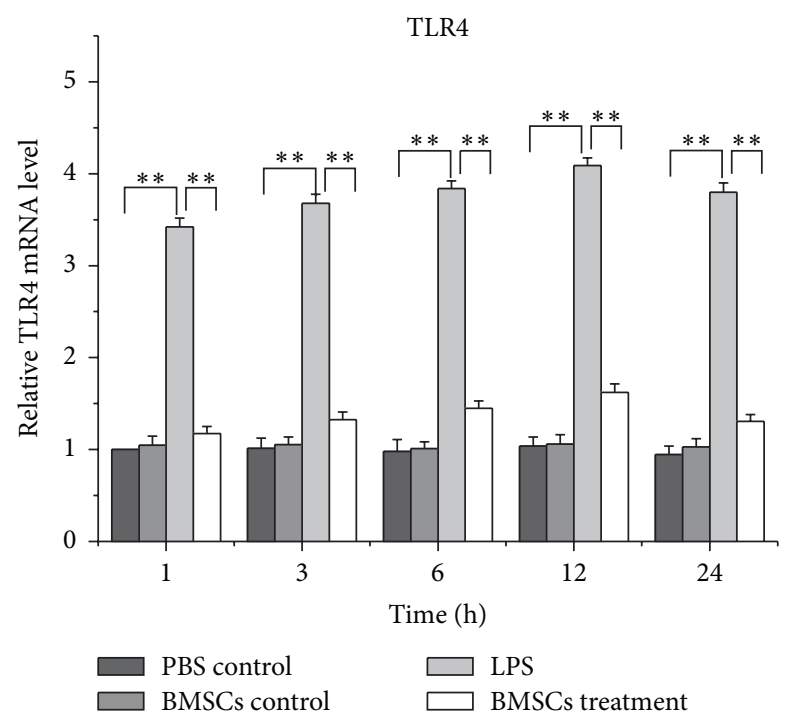

(D)

(b)

FIGURE 3: Coculture with BMSCs attenuates the activity of LPS to induce expressions of TLR2 and TLR4 in alveolar macrophages (a) and HUVECs (b) at the mRNA level. The total RNAs were extracted from the cultured alveolar macrophages and HUVECs treated (1) PBS control, (2) BMSCs, (3) LPS, and (4) BMSCs and LPS. The expressions of TLR2 and TLR4 were determined by real-time RT-PCR. Data are expressed as the mean $\pm \mathrm{SD}$ from three experiments. ${ }^{*} P<0.05,{ }^{* *} P<0.01$.

to the experiments. Further efforts are needed to clarify this discrepancy.

TLRs play a pivotal role in defense against invading pathogens through recognizing PAMPs. It is well documented that cells use TLR2 and TLR4 to recognize PAMPs expressed by bacteria. Activation of TLR2 and TLR4 leads to activation of MAPK and NF- $\kappa$ B pathways and production of inflammatory cytokines, including IL-1, IL-6, and TNF$\alpha[20-22]$. In this study, we showed that LPS promoted expression of TLR2 and TLR4 in alveolar macrophages and HUVECs. However, how expression of TLR2 expression was increased by LPS is unknown and deserves future investigations. TNF- $\alpha$ and IL10 expressions were also affected by LPS. Furthermore, the LPS effects were diminished when the HUVECs and macrophages were cultured with BMSCs. TNF- $\alpha$ is a potent inflammatory factor and IL10 is a key anti-inflammatory cytokine. Downregulation of TNF$\alpha$ and upregulation of IL10 in HUVECs and macrophage 


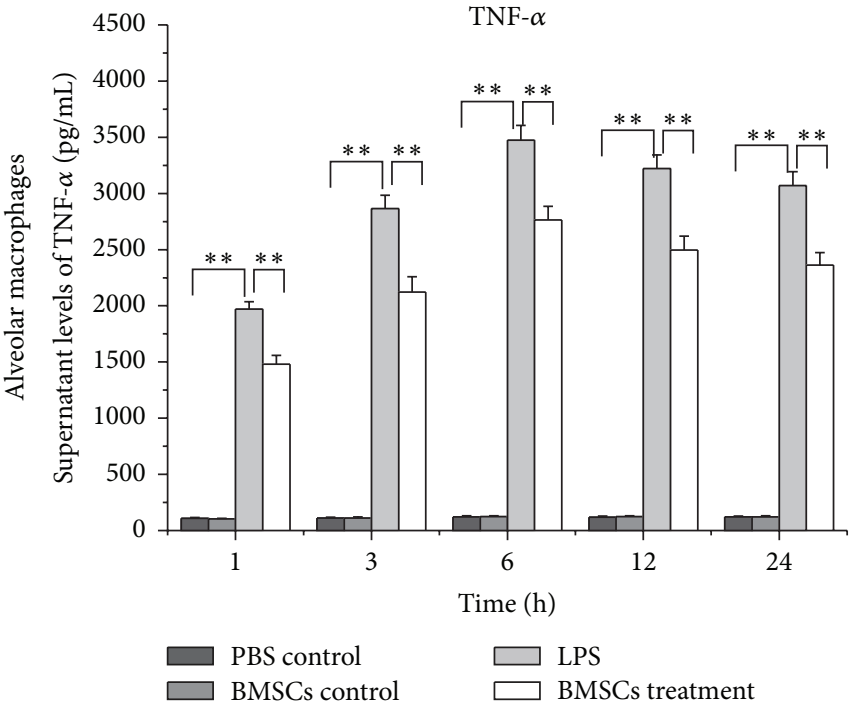

(A)

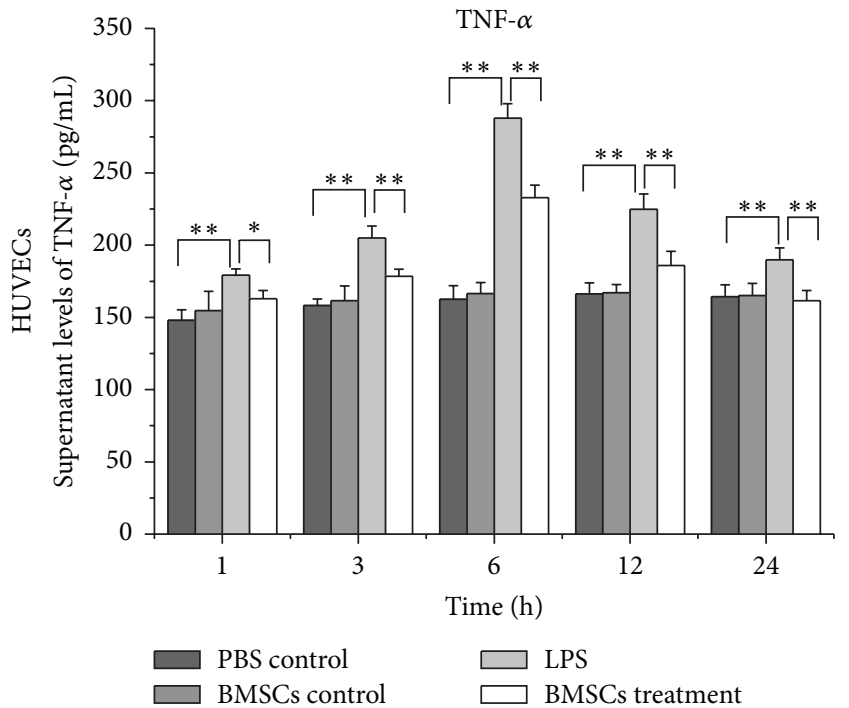

(C)

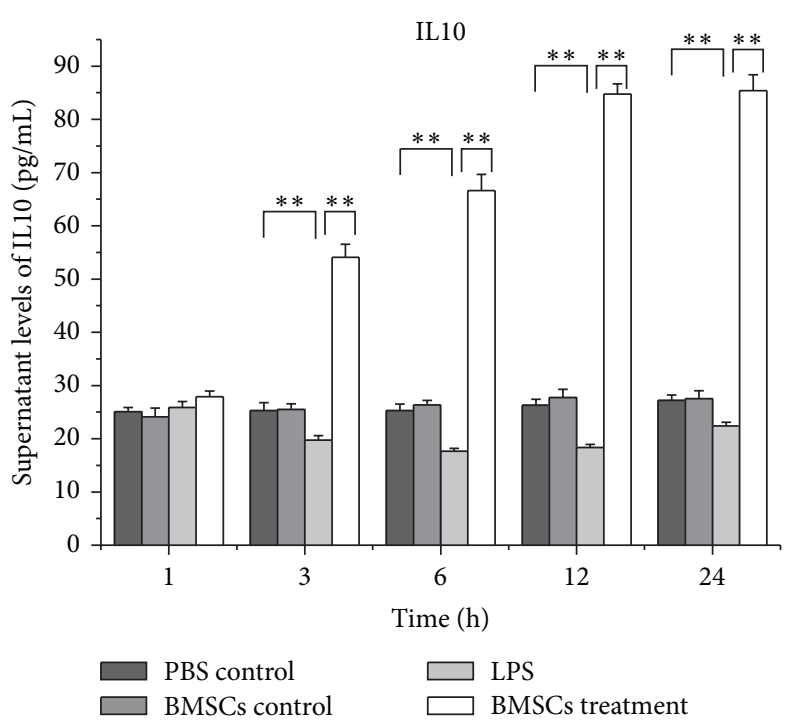

(B)

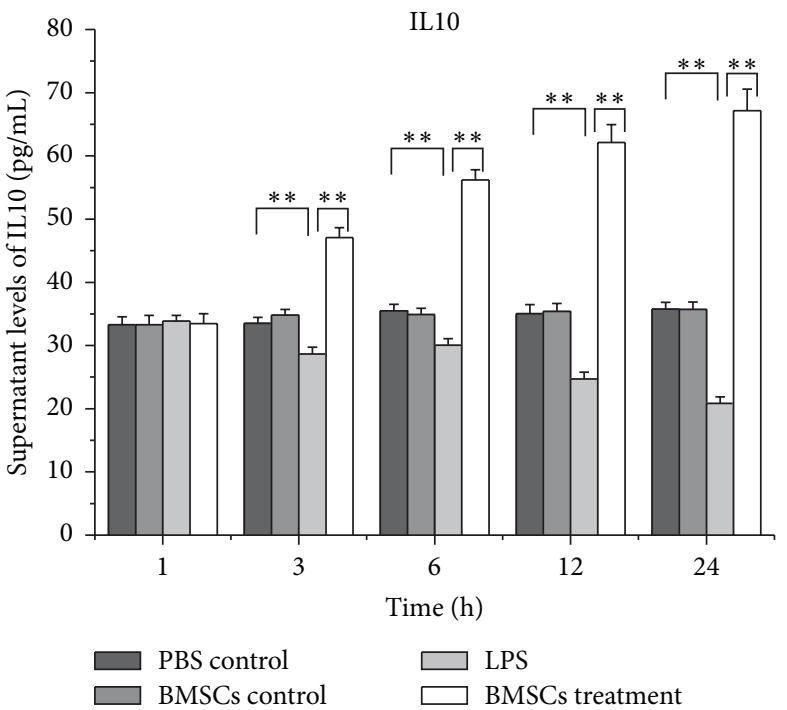

(D)

(b)

FIGURE 4: Coculture with BMSCs attenuates the activity of LPS to induce release of cytokines in alveolar macrophages (a) and HUVECs (b). Alveolar macrophages and HUVECs with or without coculture with BMSCs were treated with LPS or PBS for 1, 3, 6, 12, and 24 hours. The cell culture supernatant was then collected for cytokine analysis by ELISA. Data are expressed as the mean \pm SD from three experiments. ${ }^{*} P<0.05,{ }^{* *} P<0.01$.

indicate that the inflammatory reactions were suppressed. The results suggest that BMSCs have the ability to alleviate pathogen-induced inflammation damage in HUVECs and macrophages.

In conclusion, the results showed that BMSCs attenuated the activity of LPS on the inflammatory reaction of HUVECs and alveolar macrophages through inhibiting upregulation of TLR2, TLR4, and p65 expression, as well as downregulating TNF- $\alpha$ release and upregulating IL10 release.

\section{Abbreviations}

BMSC: Bone marrow mesenchymal stem cells

LPS: Lipopolysaccharide

TLR: Toll-like receptor

SIRS: Systemic inflammatory response syndrome

MODS: Multiple organ dysfunction syndrome

PAMP: Pathogen associated molecular patterns. 


\section{Conflict of Interests}

The authors declare that they have no conflict of interests.

\section{Authors' Contribution}

Dequan Li and Jun Fang participated in study design and coordination, analysis, and interpretation of data and supervised study. Yuanyuan Wang, Chuang Chi, and Jing Zhao performed most of the experiments and statistical analyses and drafted the paper. Other authors carried out the experiments and sample collections. All authors read and approved the final paper. Dequan Li and Cong Wang equally contributed to this paper.

\section{Acknowledgments}

The authors thank the Medical Scientific Research Platform (Wenzhou Medical University, Wenzhou, China) and the Surgery Laboratory (the First Affiliated Hospital of Wenzhou Medical University, Wenzhou, China) for their technical assistance. This work was supported by Zhejiang Province Natural Science Foundation of China (LY12H15001), Health and Family Planning Commission of Zhejiang Province (2013KYA130), Science Technology Department of Zhejiang Province (2011R50018-20), Wenzhou Municipal Science and Technology Bureau (Y20130091), and the National Natural Science Foundation of China (81171787, 31371470, and 81270761).

\section{References}

[1] J.-Z. Zhang, Z. Liu, J. Liu, J.-X. Ren, and T.-S. Sun, "Mitochondrial DNA induces inflammation and increases TLR9/NF- $\kappa \mathrm{B}$ expression in lung tissue," International Journal of Molecular Medicine, vol. 33, no. 4, pp. 817-824, 2014.

[2] T. Tsukamoto, R. S. Chanthaphavong, and H.-C. Pape, "Current theories on the pathophysiology of multiple organ failure after trauma," Injury, vol. 41, no. 1, pp. 21-26, 2010.

[3] Y. Zhao, C. Yang, H. Wang et al., "Therapeutic effects of bone marrow-derived mesenchymal stem cells on pulmonary impact injury complicated with endotoxemia in rats," International Immunopharmacology, vol. 15, no. 2, pp. 246-253, 2013.

[4] K. Tang, Y. Xiao, D. Liu et al., "Autografting of bone marrow mesenchymal stem cells alleviates streptozotocin-induced diabetes in miniature pigs: real-time tracing with MRI in vivo," International Journal of Molecular Medicine, vol. 33, no. 6, pp. 1469-1476, 2014.

[5] S. Shin, Y. Kim, S. Jeong et al., "The therapeutic effect of human adult stem cells derived from adipose tissue in endotoxemic rat model," International Journal of Medical Sciences, vol. 10, no. 1, pp. 8-18, 2012.

[6] K. Németh, A. Leelahavanichkul, P. S. T. Yuen et al., "Bone marrow stromal cells attenuate sepsis via prostaglandin $\mathrm{E}_{2}$ dependent reprogramming of host macrophages to increase their interleukin-10 production," Nature Medicine, vol. 15, no. 1, pp. 42-49, 2009.
[7] N. Kusadasi and A. B. J. Groeneveld, "A perspective on mesenchymal stromal cell transplantation in the treatment of sepsis," Shock, vol. 40, no. 5, pp. 352-357, 2013.

[8] S. R. R. Hall, K. Tsoyi, B. Ith et al., "Mesenchymal stromal cells improve survival during sepsis in the absence of heme oxygenase-1: the importance of neutrophils," STEM CELLS, vol. 31, no. 2, pp. 397-407, 2013.

[9] J. S. Elman, M. Li, F. Wang, J. M. Gimble, and B. Parekkadan, "A comparison of adipose and bone marrow-derived mesenchymal stromal cell secreted factors in the treatment of systemic inflammation," Journal of Inflammation, vol. 11, no. 1, p. 1, 2014.

[10] H. Yagi, A. Soto-Gutierrez, Y. Kitagawa, A. W. Tilles, R. G. Tompkins, and M. L. Yarmush, "Bone marrow mesenchymal stromal cells attenuate organ injury induced by LPS and burn," Cell Transplantation, vol. 19, no. 6-7, pp. 823-830, 2010.

[11] D. Togbe, S. Schnyder-Candrian, B. Schnyder et al., "Toll-like receptor and tumour necrosis factor dependent endotoxininduced acute lung injury," International Journal of Experimental Pathology, vol. 88, no. 6, pp. 387-391, 2007.

[12] W. Ren, Z. Wang, F. Hua, and L. Zhu, "Plasminogen activator inhibitor-1 regulates LPS-induced TLR4/MD-2 pathway activation and inflammation in alveolar macrophages," Inflammation, vol. 38, no. 1, pp. 384-393, 2015.

[13] K. Nemeth, B. Mayer, and E. Mezey, "Modulation of bone marrow stromal cell functions in infectious diseases by toll-like receptor ligands," Journal of Molecular Medicine, vol. 88, no. 1, pp. 5-10, 2010.

[14] N. V. Gorbunov, B. R. Garrison, D. P. McDaniel et al., "Adaptive redox response of mesenchymal stromal cells to stimulation with lipopolysaccharide inflammagen: mechanisms of remodeling of tissue barriers in sepsis," Oxidative Medicine and Cellular Longevity, vol. 2013, Article ID 186795, 16 pages, 2013.

[15] S. Jesic, A. Jotic, N. Tomanovic, M. Zivkovic, A. Kolakovic, and A. Stankovic, "Expression of toll-like receptors 2, 4 and nuclear factor kappa B in mucosal lesions of human otitis: pattern and relationship in a clinical immunohistochemical study," Annals of Otology, Rhinology and Laryngology, vol. 123, no. 6, pp. 434441, 2014

[16] Y.-H. Zheng, W. Xiong, K. Su, S.-J. Kuang, and Z.-G. Zhang, "Multilineage differentiation of human bone marrow mesenchymal stem cells in vitro and in vivo," Experimental and Therapeutic Medicine, vol. 5, no. 6, pp. 1576-1580, 2013.

[17] J. L. Shelton, L. Wang, G. Cepinskas et al., "Albumin leak across human pulmonary microvascular vs. umbilical vein endothelial cells under septic conditions," Microvascular Research, vol. 71, no. 1, pp. 40-47, 2006.

[18] Z. Zhou, F. Guo, Y. Dou, J. Tang, and J. Huan, "Guanine nucleotide exchange factor-H1 signaling is involved in lipopolysaccharide-induced endothelial barrier dysfunction," Surgery, vol. 154, no. 3, pp. 621-631, 2013.

[19] J.-S. Bae, W. Lee, and A. R. Rezaie, "Polyphosphate elicits pro-inflammatory responses that are counteracted by activated protein C in both cellular and animal models," Journal of Thrombosis and Haemostasis, vol. 10, no. 6, pp. 1145-1151, 2012.

[20] Z. Tianzhu and W. Shumin, "Esculin inhibits the inflammation of LPS-Induced acute lung injury in mice via regulation of TLR/NF- $\kappa$ B pathways," Inflammation, vol. 38 , no. 4, pp. 15291536, 2015. 
[21] Q. S. Liu, Z. W. Cheng, J. G. Xiong, S. Cheng, X. F. He, and X. C. Li, "Erythropoietin pretreatment exerts anti-inflammatory effects in hepatic ischemia/reperfusion-injured rats via suppression of the TLR2/NF- $\kappa$ B pathway," Transplantation Proceedings, vol. 47, no. 2, pp. 283-289, 2015.

[22] Y. Chen, T. Liu, P. Langford et al., "Haemophilus parasuis induces activation of NF- $\kappa \mathrm{B}$ and MAP kinase signaling pathways mediated by toll-like receptors," Molecular Immunology, vol. 65, no. 2, pp. 360-366, 2015. 


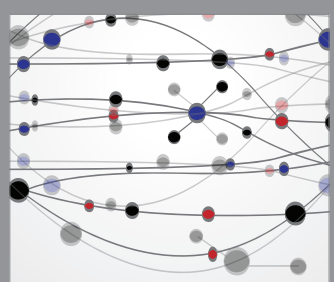

The Scientific World Journal
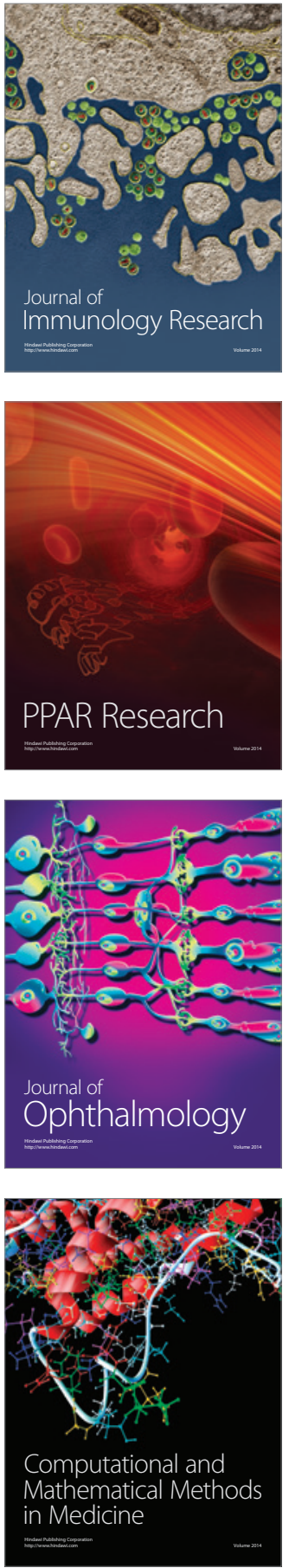

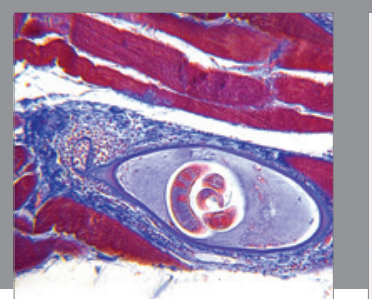

Gastroenterology Research and Practice

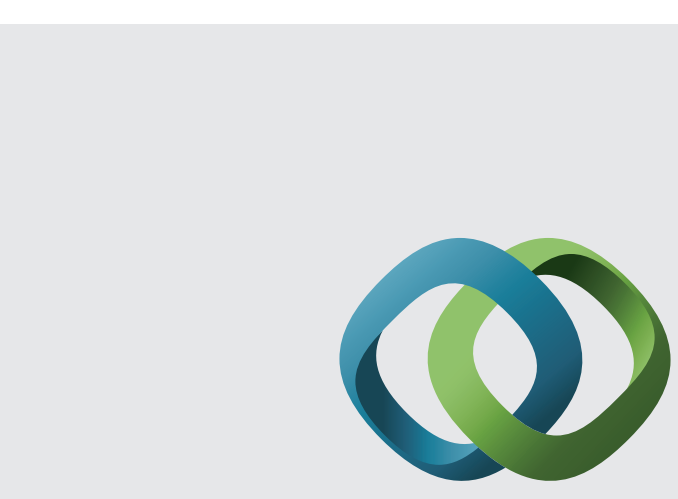

\section{Hindawi}

Submit your manuscripts at

http://www.hindawi.com
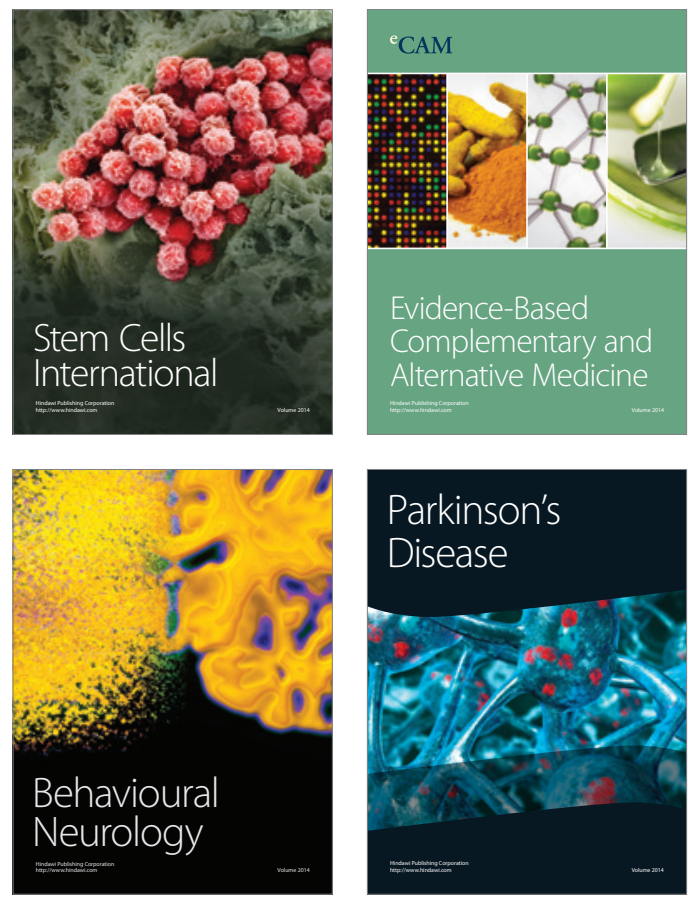
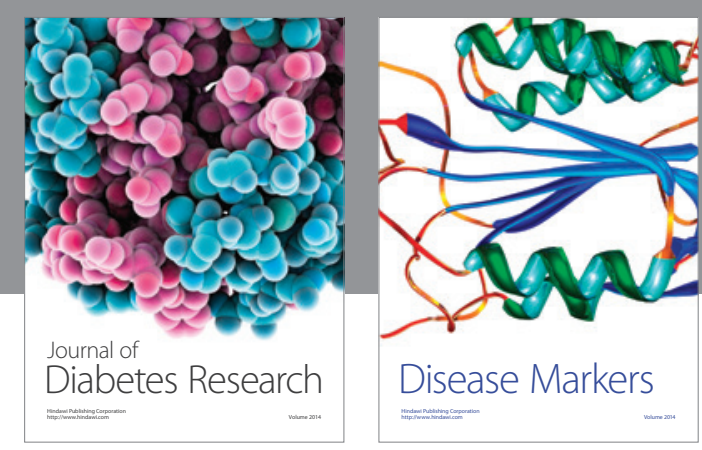

Disease Markers
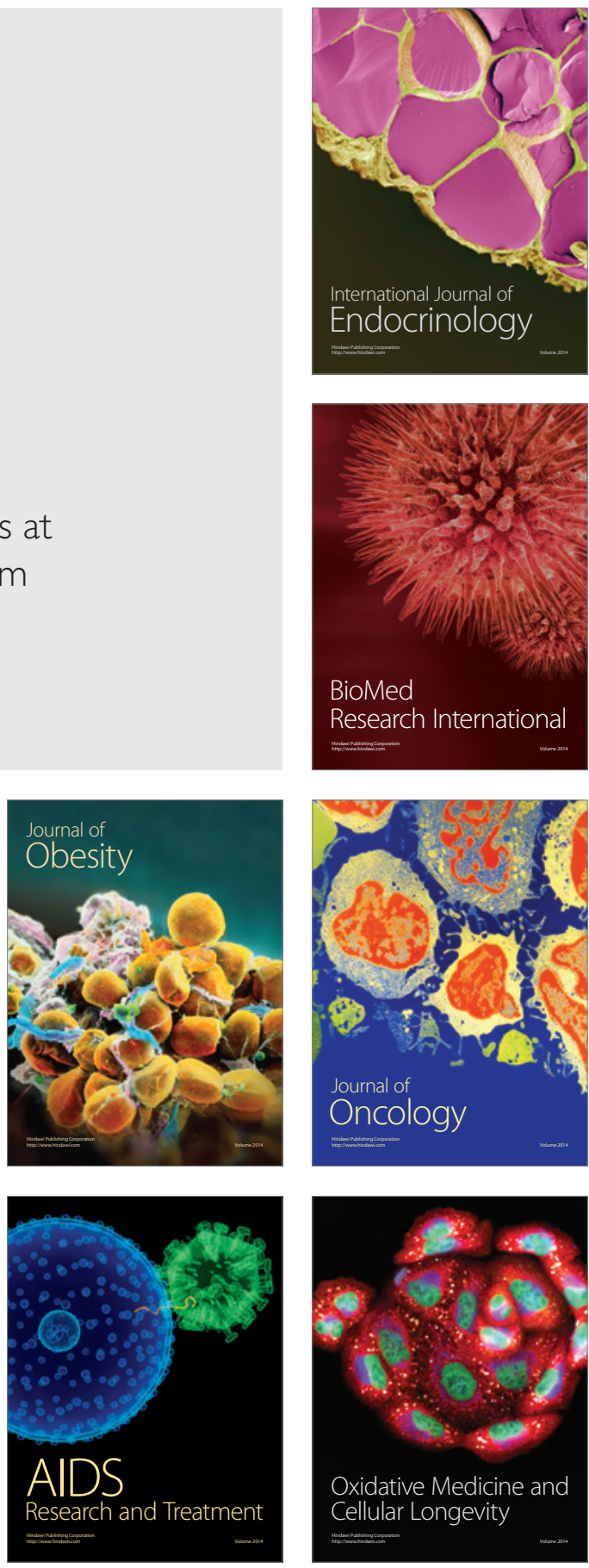